\title{
A Diagnostic Dilemma of a Pancreatic Cyst in a 12-Year Old Child
}

\author{
Wilkinson B. Gacayan MD and Faisal K. Romancap MD FPCS* \\ Department of Surgery, Cotabato Regional and Medical Center, Philippines \\ *Corresponding Author: Faisal K. Romancap MD FPCS, Department of \\ Surgery, Cotabato Regional and Medical Center, Philippines. \\ DOI: $10.31080 /$ ASGIS.2020.03.0112
}

Received: December 31, 2019

Published: January 23, 2020

(C) All rights are reserved by Wilkinson B.

Gacayan and Faisal K. Romancap.

\begin{abstract}
We present a 12-year old child who was admitted at the Emergency Department due to vague abdominal pain at the right upper quadrant and epigastric area with associated palpable mass and loose, watery stools. Initial workup included an abdominal ultrasound that revealed a hepatic abscess in the right lobe. With the presence of Entamoeba histolytica cyst on her stool exam, she was initially treated with metronidazole and antibiotic for amoebic liver abscess. Two weeks after treatment completion, severe abdominal pain recurred hence, she was re-admitted. An abdominal CT scan was requested that showed a large pancreatic head cyst. Because a cystic neoplasm cannot be ruled out, she was scheduled for intra-operative frozen section biopsy of the cyst wall before a possible excision or a roux-en-y cystojejunostomy. Frozen section biopsy of the cyst wall showed an epithelial lining within the cyst wall and subsequently a complete excision of the pancreatic head cyst was then performed. The unusual occurrence of the aforementioned condition in a young age group as well as the diagnostic and therapeutic dilemma in this case are therefore presented and discussed. Keywords: Cystic Neoplasm of the Pancreas; Pancreatic Pseudocyst; Ultrasonography; Computed Tomography; Hepatic Abscess
\end{abstract}

\section{Introduction}

Cystic neoplasm of the pancreas is considered as a rare disease entity before the advent of diagnostic modalities. It has become a well-defined radiographic entity during the last decade [1]. With the aid of these diagnostic modalities, these cysts are being increasingly identified however, majority are interpreted radiographically as pancreatic pseudocyst, being the most common benign cyst in $85 \%$ of all cystic pancreatic lesions [1]. Knowing that cystic neoplasms of the pancreas particularly the mucinous types have significant malignant potential, its early diagnosis therefore requires a high index of suspicion. Otherwise, misdiagnosis will mean mistreatment, and delay and neglect of the chance to cure a preventable and dreadful risk of pancreatic cancer [2], especially with its increasing incidence proportional with age in mucinous types [3].

\section{Case Presentation}

We present a case of a 12-year old female who was previously admitted a month prior due to intermittent, vague abdominal pain in the right upper quadrant and epigastric area associated with palpable mass and loose, watery stools. There was no jaundice, fever, and vomiting. Pertinent workup done include stool exam positive for Entamoeba histolytica cyst and an abdominal ultrasound that revealed hepatic abscess in the right lobe. She was managed as amebic liver abscess and was discharged improved. She was then re-admitted after two weeks for the same complaint of abdominal pain. Hence further workup were requested that include a normal
WBC (7 $\left.\times 10^{9} / \mathrm{L}\right)$, and three-times elevated serum amylase (310 IU/L), and lipase (380 IU/L). An abdominal CT scan was also requested and showed highly suggestive of a pancreatic pseudocyst (Figure 1). With the new findings- an elevated amylase and lipase and a pancreatic cyst on the CT scan, it was clear it was not a liver abscess but a pancreatic cyst that can either be inflammatory or neoplastic. She was able to tolerate full diet in two days, abdominal pain was resolved with analgesics, and was eventually discharged improved with the contemplated surgical plan explained to the parents and family members.

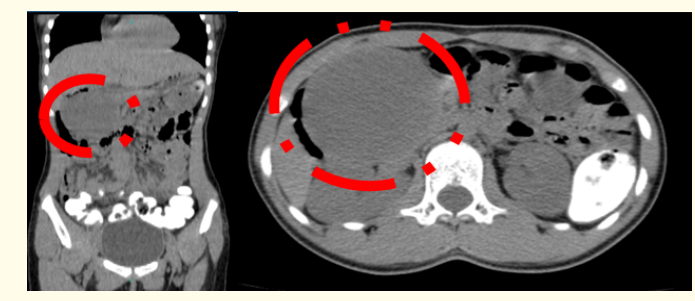

Figure 1: Coronal and axial CT without contrast showed a $6.9 \mathrm{~cm}$ widest diameter pancreatic head cyst.

The working diagnosis at this point was a pancreatic pseudocyst however, there was no clear common inciting factor that would cause pancreatitis in her age group like a gallstone or trauma to the abdomen. Idiopathic or autoimmune pancreatitis is also rare in pediatric patients. Hence, a neoplastic pancreatic cyst was not 
excluded along with other chronic sequelae of chronic pancreatitis such as walled-off pancreatic necrosis (WOPN).

Knowing that majority of the pancreatic pseudocysts communicate with the pancreatic duct, the cyst contents can drain and hence will resolve with no sequelae. Though a third of cases will have ductal disruption, these pancreatic pseudocysts can persist after six weeks. The plan of the service then was to repeat the abdominal CT scan after 6- 8 weeks. The abdominal CT scan was repeated after six weeks and showed similar findings compared to the previous. With a hepatobiliary and pediatric surgeon, we reviewed again the abdominal CT scan and showed suspicious thin septation and mural nodules (Figure 2). We also requested for tumor marker CEA $(<0.50 \mathrm{ng} / \mathrm{mL})$. Thus, we did not settle for a benign diagnosis and decided a frozen section biopsy should be done to differentiate pancreatic pseudocyst from cystic neoplasm.

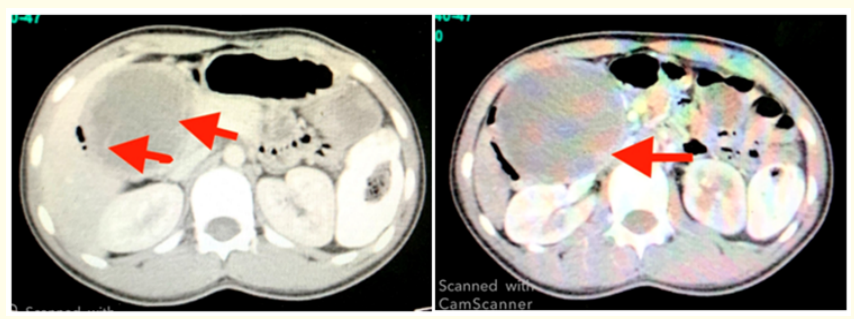

Figure 2: Suspicious thin septation and mural nodules.

Patient was subsequently admitted for elective surgery with low risk stratification for surgery. Preoperative diagnosis was a pancreatic pseudocyst versus a cystic neoplasm of the pancreatic head and the final surgical plan was exploratory laparotomy, frozen section biopsy of the cyst wall. A roux-en-y cystojejunostomy will be performed if the biopsy will show absence of epithelial cells or a complete excision if there is presence of epithelial or malignant cells.

After general anesthesia was induced with the patient in supine position, an upper midline incision was done. Intraoperative findings showed neither adhesions nor edema in the affected visceral tissues. There was no ascites, liver masses, and peritoneal implants or carcinomatosis. After opening the lesser sac, a $10 \times 8 \mathrm{~cm}$ cystic mass was seen in the pancreatic head (Figure 3). We incised elliptically a part of the cyst wall anteriorly and aspirated $10 \mathrm{~mL}$ of the serosanguinous cyst contents and sent for frozen section biopsy and cell cytology. Biopsy showed epithelial-lined cyst with mucinous fluid content (Figure 4). We conferred with the pathologist and concluded it was a mucinous cystadenoma of the head of the pancreas and then proceeded with complete excision of the cyst.

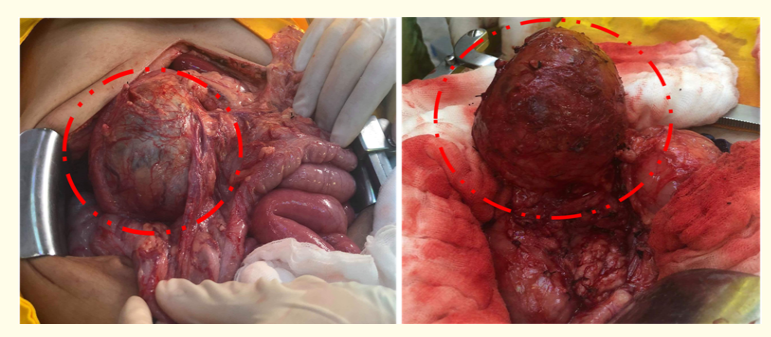

Figure 3: Intraoperative findings- cystic mass at the head of the pancreas with distinct wall and with absence of edema and adhesions.

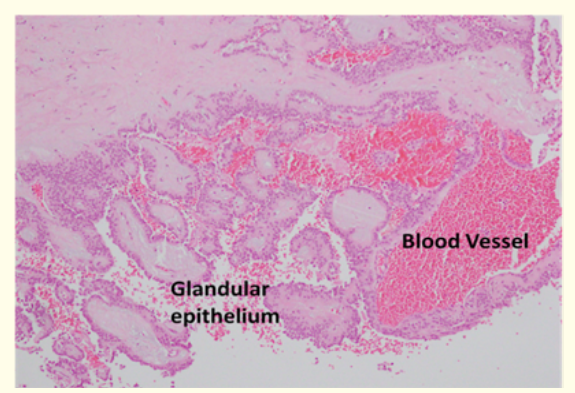

Figure 4a: Fibrous wall with epithelial cells.

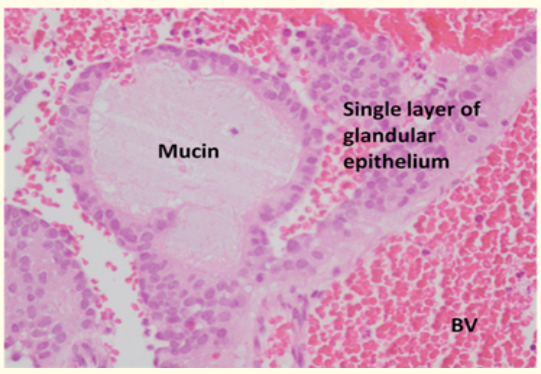

Figure 4b: Single layer of glandular epithelium with mucin (BV- blood vessel).

\section{Discussion}

Pancreatic pseudocysts are still the most common cysts of the pancreas in $85 \%$ of cases [1]. The diagnosis of pancreatic pseudocyst almost always is preceded by a history of acute pancreatitis 4 weeks prior with known causes such as gallstone, alcohol intake, abdominal trauma, hyperlipidemia, and idiopathic [1]. All these risk factors for acute pancreatitis were absent in the case.

Mucinous cystic neoplasm of the pancreas are mucin-producing cystic tumors of the pancreas that lack communication with the pancreatic duct and contain mucin-producing columnar epithelium [1]. It has a $16.1 \%$ risk of malignant potential in a cumulative span of 15- 20 years after diagnosis [4]. It is common in female in the fourth decade of life and sometimes, there is tumor degeneration and signs of metastasis [1]. Reported incidence in pediatric patients in a study is only 38 cases in children under the age of 2 years 
[5]. Th case presented is first in the Institution. Among other types of cystic neoplasms of the pancreas that are also mucin-producing and have the highest malignant potential (60\%) are intraductal papillary mucinous neoplasms (IPMNs). Other types that are nonmucin producing and have the least malignant potential are serous cystadenoma and solid pseudopapillary tumor of the pancreas [1].

Diagnosis and exclusion of cystic neoplasms of the pancreas requires high index of suspicion. Figure 5 shows the summary and algorithm in the diagnosis and treatment of the two different disease entities as shown in the case presented.

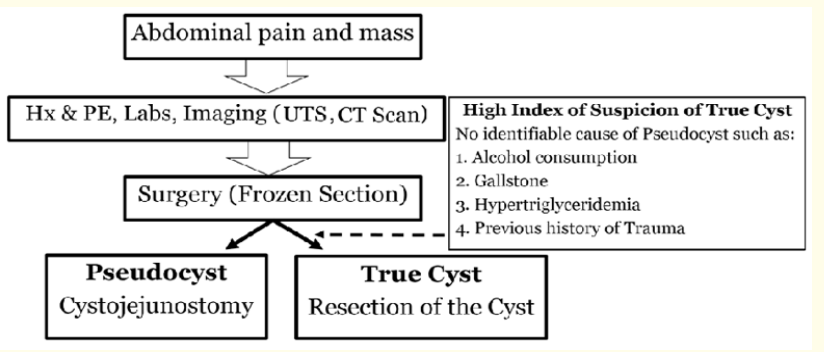

Figure 5: Algorithm in the diagnosis and management of pancreatic cysts.

\section{Conclusion}

When in doubt of diagnosis, it is always prudent to biopsy the wall of the cyst of the pancreas to make sure we would not end up draining the cyst contents of a cystic neoplasm into the small bowel or jejunum leading to the spread of the potential presence of malignant cells and converting the preventable disease into uncurable one. In addition, early diagnosis and excision of the cystic neoplasm of the pancreas is essential in the prevention of pancreatic cancer, which majority are unfortunately diagnosed in the advanced stage already.

\section{Conflict of Interest}

None.

\section{Bibliography}

1. Jarnagin William R., et al. Blumgart's Surgery of the Liver Pancreas and Biliary Tract. 6th Ed. Philadelphia, USA. Elsevier, Inc., (2017).

2. Berger Nathan A. "New light on the pancreatic cyst conundrum". Translational Cancer Research 7 (2018): S545-S548.

3. de Jong K., et al. "High prevalence of pancreatic cysts detected by screening magnetic resonance imaging examinations". Clinical Gastroenterology and Hepatology 8.9 (2010): 806-811.

4. Keane MG., et al. "Risk of Malignancy in resected pancreatic mucinous cyst neoplasms". British Journal of Surgery 105.428 (2018).
5. Gentimi FE., et al. "Pancreatic cystic lesion in an infant". Journal of Indian Association of Pediatric Surgeons 16. (2011): 72-74.

\section{Assets from publication with us}

- Prompt Acknowledgement after receiving the article

- Thorough Double blinded peer review

- Rapid Publication

- Issue of Publication Certificate

- High visibility of your Published work

Website: www.actascientific.com

Submit Article: www.actascientific.com/submission.php Email us: editor@actascientific.com

Contact us: +91 9182824667 